Astrophysics, Vol. 53, No. 4, 2010

\title{
ISOLATED DWARF GALAXIES IN THE LOCAL SUPERCLUSTER AND ITS SURROUNDINGS
}

\author{
V. E. Karachentseva ${ }^{1}$, I. D. Karachentsev², and M. E. Sharina² \\ $\left({ }^{1}\right)$ Main Astronomical Observatory, National Academy of Sciences of Ukraine, Ukraine; e-mail: \\ valkarach@gmail.com \\ ${ }^{(2)}$ Special Astrophysical Observatory, Russian Academy of Sciences; e-mail: ikar@sao.ru
}

\begin{abstract}
We present a list of 75 isolated late-type dwarf galaxies which have no neighbors with a relative radial velocity difference of less than $500 \mathrm{~km} / \mathrm{s}$ or projected separations within $500 \mathrm{kpc}$. These were 'selected from $\sim 2000$ dwarf galaxies with radial velocities $V_{L G}<3500 \mathrm{~km} / \mathrm{s}$ within the volume of the Local supercluster. In terms of their sizes, luminosities, and the amplitudes of their internal motions, the isolated late-type dwarfs do not differ significantly from gas-rich dwarf galaxies in groups and clusters. However, the median mass of neutral hydrogen per unit luminosity for the isolated dwarf galaxies is two times more than that for the late-type galaxies in groups. We have also identified 10 presumably isolated spheroidal dwarf galaxies. The detection of isolated dwarf galaxies populated 'exclusively by old stars is of great interest for modern cosmological scenarios of galaxy formation. Keywords: galaxies: dwarf galaxies
\end{abstract}

\section{Introduction}

Isolated galaxies are studied more and more actively since recent years. (See, for example, the 'Proceedings of the Conference: "Galaxies in Isolation: Exploring nature vs. Nurture" [1]). Studies ' of galaxies in low density regions allow to investigate the influence of environments on star formation processes and the evolution of galaxies, and to test various models of the origin of galaxies. Isolated dwarf galaxies, including those with low surface brightness, are of special interest for these problems.

The typical characteristics of dwarf galaxies (linear sizes of a few kpc, absolute $B$ magnitudes fainter than $-16.5^{m}$, mean surface brightness $\left\langle S B>\right.$ fainter than $25 \mathrm{~B} \mathrm{mag} / \operatorname{arcsec}^{2}$ ) [2] explain the difficulty of detecting them at large distances. Thus, dwarf galaxies are poorly represented in catalogs limited by apparent magnitude (CGCG [3], $\leq 15.7^{m}$ ) or by angular diameter, $a \geq 1^{\prime}$ (UGC [4], ESO [5]). Most objects in the UGC catalog that have been classified as "dwarfs" by their low surface brightness turned out to be ordinary spiral galaxies after their radial velocities were measured. The relative number of true dwarf galaxies in the UGC [4] is only $2 \%$. There are also few dwarf galaxies in the catalog of isolated galaxies compiled by Karachentseva [6] on the basis of the CGCG catalog. Detection of low surface brightness dwarf galaxies is an extremely time-consuming task.

The situation regarding the representation of dwarf systems changes drastically when the sample of galaxies is limited by distance, rather than flux. Thus, the Catalog of Neighboring Galaxies [2] includes 451 objects in the Local volume with distances of up to $10 \mathrm{Mpc}$; of these about $85 \%$ of the galaxies are dwarf systems, mostly with low and very low surface brightness. A determination of the so-called "tidal index" [2] for each galaxy "i" in the Local volume, 


$$
\Theta=\max \left[\log \left(M_{k} / D_{i k}^{3}\right)\right], \quad k=1,2, \ldots
$$

where $M_{k}$ is the mass and $D_{i k}$ is the mutual spatial distances of neighboring galaxies, has shown that about half the population of the Local volume occurs in groups of varying multiplicities. The rest of them are gravitationally unbound with a negative tidal index $(\Theta<0)$ and can be treated as objects in the general field. The most isolated of them, with $\Theta<-2$, turned out to be dwarf systems almost exclusively (16 out of 18 ).

A similar approach for identifying isolated galaxies has been developed in [7]. A bounding algorithm was applied to a sample of 10914 galaxies with radial velocities in the Local Group frame, $V_{L G}<3500 \mathrm{~km} / \mathrm{s}$. After pairs, triplets, and groups had been identified [8-10], roughly $46 \%$ of the remaining galaxies appeared to be unclusterized or, as they are often called, field galaxies. A more rigorous criterion of isolation, in particular the criterion [6], left only about 500 very isolated galaxies in this volume [11]. Roughly $13 \%$ of them are objects with low surface brightnesses.

An average relative number of dwarf galaxies with respect to normal ones is larger in the Local volume $\left(V_{L G}<550 \mathrm{~km} / \mathrm{s}\right)$, than in the Local supercluster $\left(V_{L G}<3500 \mathrm{~km} / \mathrm{s}\right)$. This observational selection effect is related both to the obvious decrease in the number of detected faintest dwarf systems with increasing distance, and to the fact that less attention has been paid to searches for dwarfs in the field than in the well-known nearby groups and clusters of galaxies. Nevertheless, the overall distribution of dwarf galaxies in the Local supercluster and its surroundings has been determined quite reliably up to the date. They are concentrated mainly in the Virgo and Fornax clusters and in systems having different populations, with spheroidal dwarfs located in the dense central parts and late-type dwarfs, in the more rarefied peripheral regions of groups and clusters.

This paper is the result of a search for fairly isolated dwarf galaxies of different morphological types within the volume $V_{L G}<3500 \mathrm{~km} / \mathrm{s}$. We have examined a combined sample of known field dwarf galaxies and have attempted to identify the most isolated objects within that sample. While not claiming statistical completeness of the sample, we hope to draw the attention of observers to these objects and to compare the characteristics of dwarf galaxies whose isolation has been established by various criteria.

\section{Initial data and sampling criteria}

From the complete set of known dwarf galaxies within the volume $V_{L G}<3500 \mathrm{~km} / \mathrm{s}$, we have complied an overall summary of candidate galaxies which do not enter visually in the Virgo and Fornax clusters, or known groups, and are not satellites of normal galaxies. The following sources were used for this purpose:

(1) A catalog of 1500 low surface brightness dwarf galaxies (LSB) $(<S B>$ fainter than $24.5 B$ mag/ $\left.\operatorname{arcsec}^{2}\right)[12]$. This catalog was the first attempt to gather, test, and generalize the results of searches for and observations of dwarf galaxies in articles published by various authors up to 1987. This catalog covers the entire sky; its average depth is $\sim 1500 \mathrm{~km} / \mathrm{s}$ and roughly 500 objects appear in the common field.

(2) New lists of LSB dwarf galaxies and articles about observations of these objects published after 1987, in particular searches for dwarf galaxies in the general field [13-16], HI-observations of the UGC dwarfs $[17,18]$, and several others. Out of this set of data we verified roughly 250 objects. 
(3) Articles presenting the results of continuous systematic searches for dwarf galaxies in the photographic sky surveys POSS-II (KK, KKR, KKH) [19-21], ESO-SERC (KKs) [22] and ESO SERC-J (KKSG)[23] the "5 lists", with a total number $N \sim 600$. Observations of these objects in the HI $21 \mathrm{~cm}$ line [21-27] indicated a fairly high level of detection, up to $60 \%$. Roughly 300 objects were verified from these sources.

We excluded from the lists of the candidates the objects of our Galaxy: planetary nebulae, reflecting nebulae, cirri, and also dwarf galaxies with high surface brightness. Galaxies satisfying the following conditions were chosen for further testing:

(1) objects which have radial velocities with respect to the center of the Local group within the range $500<V_{L G}<3500 \mathrm{~km} / \mathrm{s}$ and do not belong to groups and clusters;

(2) dwarf galaxies found outside the zone of strong Galactic absorption, i.e., has $A_{B}<1^{m}$.

We did not examine dwarf galaxies within the Local volume, since their properties have been summarized in the CNG catalog [2];

After refinement of the coordinates, verification of mutual identifications in the lists, and elimination of common objects independently included in different sources, we obtained a refined list of 274 late-type dwarf galaxies with measured radial velocities. Each of them was then tested according to the criterion of isolation. Here, we consider a dwarf galaxy "1" to be isolated if it has no neighbors "i" with relative radial velocities $\left|V_{1 i}\right|<500 \mathrm{~km} / \mathrm{s}$ and projected distances $R_{p}<500 \mathrm{kpc}$.

We use data on the coordinates and radial velocities of the surrounding galaxies from the NED database, using it to determine the distances to nearest neighbors. No limits were imposed on the magnitude of the neighboring galaxies. The sample of very isolated galaxies in the Local volume and their main characteristics are discussed in section 5. We checked the dwarf spheroidal galaxies without radial velocity estimates separately (section 4). Seventy five of the 274 late-type dwarf galaxies (i.e. 27\%) satisfied the isolation criterion. Of these 15 have no nearest neighbors out to a distance of $1 \mathrm{Mpc}$.

\section{List and characteristics of isolated late-type dwarf galax- ies}

Table 1 lists some of the observational data for the late type isolated dwarf galaxies. These data are mainly taken from the HyperLEDA database, and when they are lacking, from the NED database, or from original articles. In calculating the main optical and HI characteristics, we have used the computational scheme of [2] with the Hubble constant taken to be $H_{0}=73 \mathrm{~km} / \mathrm{s} / \mathrm{Mpc}$. Columns $1-$ 10 of Table 1 list the following: (1) the galaxy name; (2) the equatorial coordinates at epoch J2000.0; (3) the morphological type determined by us from images of the galaxies on the sky surveys POSS II, ESO/ SERC, DSS-1, red, blue, and, where possible, SDSS (in column 3 we omit the letter "d", for dwarf). In this paper we have divided the low surface brightness dwarf galaxies in accordance with the classification of [12]: Sm - galaxies with a disrupted spiral structure, but with a distinct center and, in some cases, with residual signs of a bar; Im — diffuse galaxies with "magellanic" features, randomly positioned blue knots ; Ir — diffuse galaxies, also of an irregular shape, but without visible blue knots ; and, Sph — diffuse galaxies with a regular circular or elliptical shape.

(4) The apparent B magnitude taken from HyperLEDA and, in some cases, from NED or original papers. Note that the error in the estimated stellar magnitude for these dwarfs can be on the order of $0.5^{m}$ or worse. (5) Radial velocity in $\mathrm{km} / \mathrm{s}$ reduced to the centroid of the Local group with the apex 
parameters accepted in NED. (6) The maximum value of the rotation velocity in $\mathrm{km} / \mathrm{s}$, corrected for the inclination, taken from HyperLEDA. (7) The absolute B magnitude corrected for Galactic and internal absorption. (8) The logarithm of the hydrogen mass, in solar units. (9) The logarithm of the hydrogen mass -to- $B$-luminosity ratio,in solar units. (10) The most isolated dwarf galaxies with no neighboring galaxies at distances to $R_{p}=1 \mathrm{Mpc}$ are indicated by an asterisk $\left(^{*}\right)$.

The last row of the table lists the median values of these characteristics of the galaxies. The following notes for Table 1 describe the shapes of some of these objects on the SDSS survey images.

\section{Candidate isolated dwarf spheroidal galaxies}

A search for presumed isolated spheroidal dwarfs in the field of the Local supercluster is a much more difficult task than searching for dwarfs of late types. By definition, spheroidal dwarfs do not contain a significant fraction of gas, so that they are not detected in the $21 \mathrm{~cm}$ line. Their optical velocities are hard to determine because of the low surface brightness of dSphs. In the general field beyond the Local volume, as well as outside the Virgo and Fornax clusters, their distances were not determined directly. Spheroidal dwarfs are often morphologically similar to irregular objects, but they have more regular shape and a smoother structure. We tried to identify candidate spheroidal dwarf objects in the general field by their morphology and then, using indirect features, to distinguish which of them are isolated.

We selected objects which have not been detected in the HI $21 \mathrm{~cm}$ line in the catalogs and lists cited in section 2, and classified as dSph and/or dSph/dIr. Our new classification using the sky surveys DSS-1 and DSS-2, and, where possible SDSS, showed that the previous search, especially in the POSS-1 survey, often gave an erroneous type estimation, and this is further confirmed by the 21 $\mathrm{cm}$ line observations. Out of the several hundred objects, we selected 107 candidates for spheroidal dwarfs or dSph/dIr without HI radial velocities. Then, using the NED database for each object, we searched for neighbors within a wide range of radial velocities, $500-3500 \mathrm{~km} / \mathrm{s}$. It turns out that most of the candidates have neighboring galaxies with radial velocities on the order of 1000-2000 $\mathrm{km} / \mathrm{s}$. Taking the same values of the radial velocities for the candidate galaxies, as well, we obtained linear dimensions and absolute magnitudes of the dwarfs which are typical of $\mathrm{dSph}$, and projected distance of $\sim 100-300 \mathrm{kpc}$ for the neighbors. Under the assumed conditions, these dwarfs cannot be regarded as isolated, although they are, most likely, within the volume of the Local supercluster.

After verification of the 107 candidates in our list of presumed isolated dwarf spheroidal galaxies, only 10 objects remained. Their main characteristics (taken from original articles [19-22] or the NED) are listed in Table 2, where the contents of the columns do not require further explanation. 
Table 1: List of isolated late-type dwarf galaxies within the Volume $V_{L G}<3500 \mathrm{~km} / \mathrm{s}$

\begin{tabular}{|c|c|c|c|c|c|c|c|c|c|}
\hline Name & RA(J2000.0)DEC & Type & $B$ & $V_{L G}$ & $V_{r}$ & $M_{B}^{c}$ & $\log \left(M_{H I}\right)$ & $\log \left(M_{H I} / L_{B}\right)$ & $*$ \\
\hline (1) & $(2)$ & (3) & (4) & $(5)$ & (6) & (7) & (8) & (9) & (10) \\
\hline ESO 12-14 & $000244.2-802048$ & Sm & 14.83 & 1757 & 44 & -17.5 & 9.81 & 0.65 & \\
\hline UGC 199 & $002051.8+125122$ & $\mathrm{Sm}$ & 17.3 & 2012 & 46 & -15.2 & 8.78 & 0.54 & \\
\hline ESO $474-25$ & 004707.5-223542 & $\mathrm{Sm}$ & 16.0 & 2896 & 57 & -17.1 & 9.12 & 0.14 & * \\
\hline UGC 672 & $010617.9+445715$ & Ir & 17.1 & 967 & 36 & -14.1 & 7.89 & 0.11 & \\
\hline ESO243-50 & 011048.8-422231 & $\mathrm{Im} / \mathrm{Ir}$ & 17.04 & 1413 & 26 & -14.4 & 8.78 & 0.86 & Th \\
\hline LSBC F683-V10 & $013031.9+112925$ & Ir & 17.51 & 1134 & - & -13.9 & - & - & \\
\hline KDG 9 & $014234.0+025546$ & Ir & 16.9 & 1882 & - & -15.3 & - & - & \\
\hline KKH 8 & $021227.4+101958$ & Ir & 16.7 & 1861 & 24 & -15.8 & 8.30 & -0.20 & \\
\hline ESO 545-2 & 021915.3-185556 & $\mathrm{Sm}$ & 14.96 & 1603 & 58 & -17.1 & 9.14 & 0.15 & \\
\hline ESO 298-33 & 022128.1-384803 & $\operatorname{Im}$ & 17.76 & 2142 & 25 & -14.7 & 8.81 & 0.79 & \\
\hline ESO 545-15 & 022557.9-194130 & Im & 16.72 & 2272 & 33 & -15.9 & 9.37 & 0.86 & \\
\hline KKH 14 & $024502.9+320942$ & Ir & 17.0 & 1748 & 45 & -15.7 & 9.27 & 0.82 & * \\
\hline UGC 2352 & $025205.4+042215$ & $\operatorname{Im}$ & 17.2 & 1866 & 35 & -15.1 & 8.86 & 0.67 & \\
\hline KDG 32 & 031856.7-103247 & S0 & 16.74 & 1898 & 42 & -15.7 & 9.04 & 0.60 & * \\
\hline AM 0333-611 & 033415.3-610548 & Im & 16.38 & 976 & 24 & -14.4 & 7.96 & 0.04 & \\
\hline HIPASS J0341+18 & $034201.7+180831$ & $\operatorname{Im}$ & 16.90 & 1369 & 18 & -15.4 & 8.37 & 0.06 & \\
\hline ESO 251-3 & $042841.2-461916$ & $\operatorname{Im}$ & 16.19 & 1191 & 31 & -14.9 & 8.32 & 0.19 & \\
\hline KK 271 & $045145.3+670932$ & $\mathrm{Ir} / \mathrm{Sph}$ & 17.7 & 1771 & - & -15.1 & 8.04 & -0.17 & * \\
\hline ESO 555-39 & 060901.1-214323 & $\mathrm{Sm}$ & 15.89 & 1535 & 59 & -16.1 & 8.84 & 0.24 & \\
\hline UKS 0616-708 & 061549.2-705342 & $\mathrm{Sm}$ & 15.5 & 1025 & 19 & -15.6 & 8.86 & 0.45 & \\
\hline KK 56 & $064311.6+363803$ & Ir & 17.9 & 2360 & 29 & -15.3 & 8.30 & 0.02 & \\
\hline UGC 3672 & $070627.5+301919$ & $\mathrm{Im} / \mathrm{Pec}$ & 16.4 & 969 & 44 & -14.5 & 9.10 & 1.12 & \\
\hline KK 58 & 070910.7-512801 & Ir & 15.38 & 772 & 17 & -15.3 & 8.27 & -0.01 & \\
\hline KK 66 & $074729.8+401116$ & Ir & 17.5 & 2949 & 31 & -15.7 & 8.85 & 0.39 & \\
\hline UGC 4117 & $075726.0+355621$ & $\operatorname{Im}$ & 15.85 & 754 & 28 & -14.4 & 8.08 & 0.15 & \\
\hline UGC 4100 & $080656.9+844515$ & Im & 16.0 & 1221 & 38 & -15.4 & 8.59 & 0.29 & * \\
\hline KKH 44 & $081638.5+692049$ & Ir & 18.2 & 1202 & 8 & -13.0 & 7.88 & 0.51 & * \\
\hline UGC 4527 & $084424.0+765505$ & Ir & 16.9 & 900 & 68 & -13.8 & 7.96 & 0.30 & \\
\hline KDG 54 & $092225.2+754557$ & $\mathrm{Im} / \mathrm{Ir}$ & 16.4 & 833 & 20 & -14.0 & 7.97 & 0.21 & \\
\hline KKH 52 & $093747.2+273404$ & $\mathrm{Im} / \mathrm{Ir}$ & 16.6 & 1513 & 15 & -15.1 & 8.45 & 0.27 & \\
\hline KDG 69 & $102852.6+664823$ & Ir & 17.4 & 1268 & 33 & -13.8 & 8.45 & 0.75 & \\
\hline UGC 5996 & $105257.4+402242$ & $\mathrm{Sm}$ & 16.8 & 1617 & 37 & -15.1 & 8.56 & 0.35 & \\
\hline UGC 6222 & $111104.5+343411$ & $\mathrm{Sm}$ & 16.2 & 1926 & 33 & -16.0 & 8.82 & 0.26 & \\
\hline KK 102 & $112258.9+192839$ & Ir & 16.45 & 3268 & 45 & -16.9 & 8.87 & -0.05 & \\
\hline KK 105 & $112924.2+460651$ & Ir & 16.6 & 1598 & 40 & -15.2 & 8.36 & 0.13 & \\
\hline UGC 6996 & $120022.7+785103$ & $\mathrm{Sm}$ & 17.1 & 2161 & 56 & -15.6 & 8.75 & 0.36 & \\
\hline KKs 48 & 120535.9-434612 & Ir & 17.62 & 2732 & 45 & -15.8 & 9.39 & 0.91 & \\
\hline KDG 89 & 121428.9-121957 & Ir & 17.25 & 1714 & 43 & -14.8 & 8.57 & 0.49 & \\
\hline UGC 7995 & $124957.7+782302$ & Ir & 16.25 & 1998 & 57 & -16.2 & 8.59 & -0.04 & * \\
\hline KK 181 & $130433.7+264627$ & $\operatorname{Im} / \operatorname{Ir}$ & 17.25 & 1914 & 36 & -14.9 & 8.77 & 0.65 & * \\
\hline KK 183 & $130642.4+180007$ & $\mathrm{Sph} / \mathrm{Ir}$ & 17.9 & 1496 & 43 & -13.8 & 8.34 & 0.67 & \\
\hline UGC 8474 & $132926.4+005412$ & $\mathrm{Sm}$ & 16.26 & 3164 & 30 & -17.0 & 8.86 & -0.11 & \\
\hline KDG 227 & $133439.7-121950$ & $\mathrm{Sm}$ & 15.59 & 1347 & 41 & -16.0 & 8.23 & -0.31 & \\
\hline LSBC D721-V10 & $135557.8+085950$ & Ir & 17.8 & 1165 & 22 & -13.3 & 7.17 & -0.32 & \\
\hline KK 226 & 135608.4-453934 & $\mathrm{Sm}$ & 15.91 & 2300 & 28 & -16.9 & 9.06 & -0.11 & \\
\hline KKR 2 & $140626.2+092133$ & $\mathrm{Ir} / \mathrm{Im}$ & 17.32 & 3213 & 34 & -16.0 & 8.76 & 0.19 & * \\
\hline
\end{tabular}




\begin{tabular}{|c|c|c|c|c|c|c|c|c|c|}
\hline$(1)$ & $(2)$ & $(3)$ & $(4)$ & $(5)$ & $(6)$ & $(7)$ & $(8)$ & $(9)$ & $(10)$ \\
\hline UGC 9123 & $141503.7+362726$ & $\mathrm{Sm}$ & 15.73 & 1951 & 62 & -16.5 & 8.77 & 0.01 & \\
\hline KKR 6 & 141703.6-013015 & $\operatorname{Im}$ & 16.93 & 1463 & 25 & -14.8 & 8.41 & 0.33 & \\
\hline KKSG 47 & 143525.4-171001 & $\mathrm{Ir}$ & 17.7 & 1447 & 54 & -14.2 & 8.92 & 1.08 & \\
\hline KKR 12 & $144624.3+141245$ & Ir & 16.6 & 1801 & 20 & -15.4 & 8.77 & 0.43 & \\
\hline UGC 9875 & $153047.3+230358$ & $\mathrm{Sm}$ & 15.83 & 2073 & 86 & -16.7 & 8.95 & 0.12 & \\
\hline UGC 9912 & $153510.5+163258$ & $\mathrm{Sm}$ & 15.56 & 1032 & 19 & -15.4 & 8.60 & 0.28 & \\
\hline KKR 21 & $153700.6+204742$ & $\mathrm{Sm} / \mathrm{Im}$ & 16.8 & 1798 & 34 & -15.4 & 8.94 & 0.62 & \\
\hline UGC 10009 & $154537.5+041057$ & $\mathrm{Ir}$ & 17.0 & 2095 & 64 & -16.0 & 8.71 & 0.15 & \\
\hline UGC 10229 & 160943.9-000654 & $\mathrm{Im}$ & 17.35 & 1522 & 44 & -14.8 & 9.15 & 1.08 & \\
\hline KKR 26 & $161644.6+160509$ & $\mathrm{Ir}$ & 17.1 & 2347 & 24 & -15.6 & 8.75 & 0.34 & $*$ \\
\hline UGC 10376 & $162250.9+652616$ & $\mathrm{Sm}$ & 16.5 & 3246 & 36 & -16.8 & 9.01 & 0.11 & \\
\hline LSBC F585-V01 & $162557.4+203934$ & $\mathrm{Ir}$ & 18.4 & 2106 & 26 & -14.2 & - & - & \\
\hline KKR 30 & $165638.5+075956$ & Ir & 17.0 & 1584 & 31 & -15.2 & 8.73 & 0.49 & $*$ \\
\hline KKR 34 & $171242.1+135428$ & Ir & 17.8 & 1640 & 37 & -14.6 & 8.69 & 0.68 & \\
\hline KKR 39 & $175900.7+215053$ & $\mathrm{Ir}$ & 18.1 & 2242 & 24 & -14.8 & 8.76 & 0.67 & \\
\hline KKR 42 & $181052.1+371453$ & $\mathrm{Ir}$ & 17.7 & 1754 & 12 & -14.4 & 8.06 & 0.14 & \\
\hline KKs 68 & $182246.5-621613$ & $\mathrm{Im}$ & 16.7 & 808 & 23 & -13.9 & 7.81 & 0.10 & \\
\hline UGC 11458 & $193506.9+695942$ & Ir & 17.5 & 1651 & 65 & -15.1 & 8.54 & 0.36 & $*$ \\
\hline KKR 48 & $195756.4+623721$ & Ir & 17.5 & 3453 & 17 & -16.2 & 8.94 & 0.29 & \\
\hline KK 249 & 202906.9-314110 & $\mathrm{Ir}$ & 15.79 & 2169 & 66 & -17.1 & 8.80 & -0.21 & \\
\hline ESO 403-36 & 215010.9-354228 & $\mathrm{Ir}$ & 16.90 & 2570 & 75 & -16.1 & 9.58 & 0.99 & \\
\hline KK 255 & 215755.8-601822 & $\mathrm{Im}$ & 14.6 & 1585 & 22 & -17.2 & 8.11 & -0.95 & \\
\hline ESO 189-21 & $220236.6-540443$ & $\mathrm{Sm}$ & 15.31 & 1644 & 28 & -16.5 & 8.94 & 0.16 & \\
\hline ESO 602-16 & 222323.9-180730 & $\mathrm{Im}$ & 15.68 & 2723 & 52 & -17.4 & 9.26 & 0.16 & \\
\hline KKR 71 & $223038.0+384355$ & $\mathrm{Ir}$ & 17.3 & 1002 & 11 & -14.0 & 8.65 & 0.91 & \\
\hline UGC 12212 & $225030.3+290818$ & $\mathrm{Sm}$ & 16.2 & 1177 & 45 & -15.1 & 8.88 & 0.66 & \\
\hline KKR 75 & $232011.2+103723$ & $\mathrm{Ir}$ & 18.0 & 1703 & 45 & -14.1 & 8.66 & 0.87 & $*$ \\
\hline UGC 12070 & $233157.3+780903$ & $\operatorname{Im}$ & 16.5 & 1724 & 48 & -16.3 & 9.08 & 0.38 & $*$ \\
\hline UGC 12771 & $234532.7+171512$ & $\mathrm{Im} / \mathrm{Ir}$ & 16.5 & 1535 & 38 & -15.4 & 8.48 & 0.17 & \\
\hline MEDIANS & & & 16.8 & 1720 & 35 & -15.3 & 8.76 & 0.30 & \\
\hline
\end{tabular}

Notes for Table 1:

ESO 545-2: wedge shaped, with blue knots;

UGC 3672: long diffuse "tail" with knots directed to the NW;

UGC 5996: distinct brightness gradient, a diffuse blue shell and several jets with knots;

UGC 6222: possibly dIr, bluish, with diffuse knots;

KK 181: blue, with diffuse "spots", without bright knots ;

UGC 8474: smooth spiral with a diffuse central part;

LSBC D721-V10: noticeable brightness gradient, a star is projected;

KKR 2: faint knots;

KKR 12: diffuse patchy knots;

KKR 6: faint knots;

UGC 9123: regular diffuse spiral arms with several blue knots;

UGC 9875: regular diffuse spiral arms with several blue knots;

UGC 9912: regular spiral, diffuse nuclear part and blue knots in the spiral arms;

KKR 21: disrupted spiral, very faint knots, a starr is projected;

UGC 10009: irregular, without a brightness gradient;

UGC 10229: knots;

KKR 26: faint blue knot;

UGC 12771: diffuse, filamentary. 
Table 2: List of presumed isolated dSph galaxies in the volume $V_{L G}<3500 \mathrm{~km} / \mathrm{s}$

\begin{tabular}{lccclcl}
\hline Name & RA (J2000.0) Dec & $a^{\prime}$ & $b / a$ & mag & $A_{B}$ & Other name \\
\hline$(1)$ & $(2)$ & $(3)$ & $(4)$ & $(5)$ & $(6)$ & $(7)$ \\
\hline KKH 9 & $021254.9+324854$ & 1.1 & 0.91 & 16.6 & 0.42 & UGC 1703 \\
KKs 5 & $031407.2-375933$ & 1.2 & 0.58 & 16.7 & 0.07 & ESO 300-20 \\
KKH 65 & $105159.2+282145$ & 0.7 & 1.00 & 17.0 & 0.08 & BTS 23 \\
KKH 66 & $110222.3+701553$ & 1.0 & 0.60 & 18.0 & 0.10 & KDG 74 \\
KKH 67 & $112303.5+211937$ & 1.2 & 0.75 & 16.8 & 0.10 & \\
KK 180 & $130429.9+174532$ & 1.4 & 0.50 & 16.7 & 0.10 & LSBC D575-8 \\
KK 227 & $135610.1+401812$ & 0.7 & 0.64 & 17. & 0.04 & \\
KKR 8 & $141914.8+030726$ & 1.3 & 0.62 & 18.5 & 0.15 & \\
KKR 9 & $142705.0+224125$ & 0.9 & 0.67 & 19 & 0.29 & \\
KK 258 & $224043.9-304800$ & 2.1 & 0.62 & 17.4 & 0.06 & ESO 468-20 \\
\hline
\end{tabular}

Notes for Table 2:

KKH 9: According to the NED, this is an elliptical dwarf dE. On the SDSS image it appears to be dSph; no neighbors within the range of $500-2500 \mathrm{~km} / \mathrm{s}$ found in a region with a radius of $120^{\prime}$.

KKs 5: Appears to be dSph on DSS-1 and dSph/dIr on DSS-red images. The object LSBG F 300-1 (dE) with $V_{L G}=1456 \mathrm{~km} / \mathrm{s}$ is at a distance of $37.4^{\prime}$ and if KKs 5 has a radial velocity close to that of LSBG F 300-1, then its linear size should be $A \sim 7 \mathrm{kpc}$, which is not typical for spheroidal dwarfs. Thus, we do not accept the calculated mutual distance $(\sim 220 \mathrm{kpc})$ and we consider KKs 5 to be an isolated object. There is also the object FCCB 035 with $V_{L G}=871 \mathrm{~km} / \mathrm{s}$ at a distance of $\sim 140 \mathrm{kpc}$. If they have similar radial velocities, then the linear diameter is $A \sim 4 \mathrm{kpc}$.

KKH 65: On the SDSS survey image it appears to be a dSph galaxy with a very low surface brightness (VLSB). Its membership in the NGC 4314 group implies an atypical linear diameter (> 4 $\mathrm{kpc})$.

KKH 66: In the DSS-red, blue, it shows up as a dwarf spheroidal galaxy. The nearest galaxy, GCGC 333-63 has $V_{L G}=1438 \mathrm{~km} / \mathrm{s}$. If the radial velocities are similar, their mutual distance is roughly $450 \mathrm{kpc}$ and the linear diameter $A \sim 5.7 \mathrm{kpc}$ (unrealistically large for $\mathrm{dSph}$ ).

KKH 67: On the SDSS survey image it shows up as a typical dSph, VLSB galaxy. The dwarf galaxy LSBC D570-7 with $V_{L G}=713 \mathrm{~km} / \mathrm{s}$ is at an angular distance of $29.8^{\prime}$. If they are a physical pair, then the linear size of KKH 67 is $A=3.5 \mathrm{kpc}$ and the projected distance is $85 \mathrm{kpc}$. We consider KKH 67 to be nominally isolated.

KK 180: On the SDSS survey image it appears as a typical dSph galaxy. If we assume that KK 180 is at the same distance as KK 173 with $V_{L G}=952 \mathrm{~km} / \mathrm{s}$, we obtain a linear diameter of $\sim 5.3$ $\mathrm{kpc}$ and a mutual distance of $\sim 320 \mathrm{kpc}$ for KK 180. If, on the other hand, KK 180 belongs to the periphery of the NGC 4826 group, then its diameter $A \sim 2.2 \mathrm{kpc}$ and its nearest neighbor, at a distance of $\sim 220 \mathrm{kpc}$, turns out to be KDG 215 with $V_{h}=419 \mathrm{~km} / \mathrm{s}$. We consider this galaxy to be nominally isolated.

KK 227: In a field of radius $120^{\prime}$ there are many galaxies with $V_{h} \sim 2300 \mathrm{~km} / \mathrm{s}$, the closest of which is NGC 5571 with $V_{L G}=2637 \mathrm{~km} / \mathrm{s}$. If KK 227 is a physical companion of NGC 5571 (at a projected distance of $117 \mathrm{kpc}$ ), then its linear diameter of $\sim 7.3 \mathrm{kpc}$ is too large for a typical dwarf spheroidal galaxy.

KKR 8: On the DSS-1 blue image it appears as a typical dSph galaxy. The nearest galaxy is 
NGC 5576 with $V_{L G}=1437 \mathrm{~km} / \mathrm{s}$ (at a projected distance of $146 \mathrm{kpc}$ ), a member of the group NGC 5666. But if KKR 8 belongs to this group, then its linear diameter $A=7.4 \mathrm{kpc}$ is too large for dSph.

KKR 9: On the DSS-1 blue image it appears as dSph, VLSB. We assume that the nearest neighbor is a member of the Local volume, DDO 187. But if these galaxies are a physical pair (107 kpc), then the linear size of KKR 9 is $A=0.6 \mathrm{kpc}$. Furthermore, within a region of radius $85^{\prime}$ there are several galaxies with radial velocities of $\sim 1100-1200 \mathrm{~km} / \mathrm{s}$; the closest of all $(390 \mathrm{kpc})$ is a magellanic-type dwarf galaxy CGCG 133-84 which seems not to be gravitationally bound with KKR 9. Note that, by its morphology, object KKR 9 is similar to the known isolated dSph galaxy KKR 25, which is located at a distance of $1.9 \mathrm{Mpc}$.

KK 258: On the DSS-1 survey image this shows up as a dSph/dIr galaxy and on DSS-red, as dSph. If we assume that the galaxy NGC 7361 with $V_{L G}=1130 \mathrm{~km} / \mathrm{s}$ (at a projected distance of $250 \mathrm{kpc}$ ) is a physical neighbor, then the linear diameter of KK 258 is equal to $11 \mathrm{kpc}$, which is unrealistic. We note that the radial velocity of KK 258 given in LEDA, $V_{h}=27246 \mathrm{~km} / \mathrm{s}$, is wrong.

Figure 1 shows the all-sky distribution of the isolated dwarf galaxies in equatorial coordinates. The solid circles indicate dwarf galaxies of later types and the open circles mark dwarf spheroidal galaxies. It appears, that the distribution of the late-type dwarfs is not entirely uniformly random. There is an indication of some excess in the number of objects in the region centered at RA $16^{h} .0$, Dec $=+15$. The median velocity of these 12 galaxies is $+1800 \mathrm{~km} / \mathrm{s}$. The position on the sky and the characteristic velocity suggest that this group is located on the distant edge of the Local Void [28].

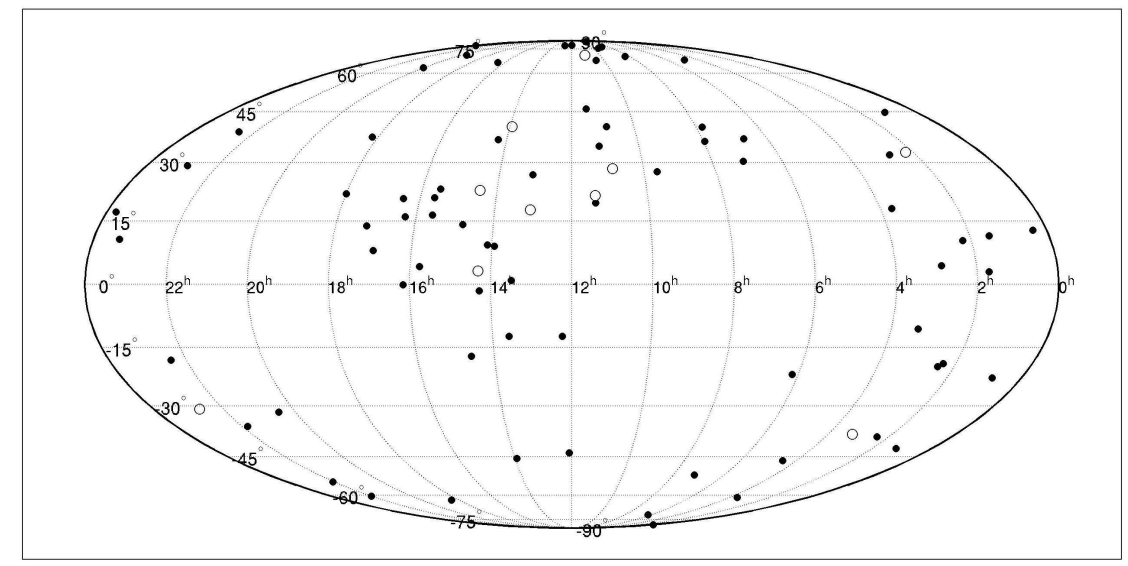

Figure 1: Distribution of isolated dwarf galaxies over the sky in equatorial coordinates. The solid circles indicate late-type dwarf galaxies and the open circles, spheroidal dwarf galaxies 
Table 3: Characteristics of the most isolated galaxies in the Local volume

\begin{tabular}{lccrrrc}
\hline Name & Type & $A_{B}$ & $V_{m}$ & $M_{B}^{c}$ & $\log M_{H I} / L$ & $\Theta$ \\
\hline UGC 288 & Im & 2.47 & 19 & -13.82 & 0.01 & -2.1 \\
And IV & Ir/Sph & 2.32 & 49 & -12.60 & 1.09 & -2.1 \\
IC 2038 & Sdpec & 2.63 & 50 & -14.42 & 0.48 & -2.2 \\
KK 49 & Im & 1.65 & 34 & -14.94 & -0.42 & -2.0 \\
ORION DW & Sm/Im & 6.19 & 81 & -17.04 & -0.14 & -2.2 \\
ESO 364-29 & Impec & 7.61 & 40 & -16.04 & -0.27 & -2.9 \\
ESO 489-56 & Impec & 0.80 & 9 & -13.07 & -0.34 & -2.1 \\
UGC 3476 & Im/Sm & 1.98 & 38 & -14.27 & 0.25 & -2.2 \\
KK 55 & Ir & 1.54 & 29 & -13.71 & -0.43 & -2.6 \\
FG 202 & Ir & 4.63 & 38 & -14.01 & 0.38 & -2.0 \\
ESO 558-11 & Im & 4.52 & 61 & -16.51 & -0.89 & -2.5 \\
UGC 3755 & Ir & 2.51 & 16 & -14.90 & -0.52 & -2.1 \\
DDO 47 & Sm/Im & 4.65 & 46 & -15.10 & 0.45 & -2.1 \\
ESO 6-1 & Sm/Im & 2.54 & - & -14.86 & - & -2.4 \\
KKH 46 & $\operatorname{Im}$ & 1.17 & 16 & -11.93 & 0.35 & -2.0 \\
ESO 564-30 & $\mathrm{Sm}$ & 3.86 & 68 & -14.27 & 0.80 & -2.0 \\
KK 246 & $\mathrm{Ir}$ & 2.14 & 25 & -12.96 & 0.41 & -2.2 \\
IC 5052 & $\mathrm{Sd}$ & 7.64 & 83 & -18.23 & -0.49 & -2.2 \\
\hline Medians & - & 2.51 & 38 & -14.34 & 0.01 & -2.1 \\
\hline Nof for Table & & & & & &
\end{tabular}

Notes for Table 3:

KK 49 and Orion is located in the region of strong Galactic

absorption $\left(A_{B}=2.46^{m}\right.$ and $3.16^{m}$,respectively);

IC 5052 is not a LSB dwarf galaxy.

\section{The most isolated galaxies in the Local volume}

As a supplement to the above lists (Tables 1 and 2), in Table 3 we list the galaxies in the Local volume which are the most isolated $(\Theta \leq-2.0)$ according to the criterion of the CNG [2]. A value of $\Theta=-2.0$ corresponds approximately to a local density of matter two orders below the mean density. The columns in the table list the following: (1) galaxy name, (2) morphological type according to our classification, (3) corrected linear size in $\mathrm{kpc}$, (4) maximum rotational velocity in $\mathrm{km} / \mathrm{s}$, (5) absolute B magnitude, corrected for Galactic and internal absorption, (6) logarithm of the hydrogen mass-to-B luminosity ratio, in solar units, and (7) the "tidal index" $\Theta$. The data in all the columns, except 2, are taken from Table 4 of the CNG. The last row of the table gives the median values of the corresponding characteristics of the galaxies. The galaxies in Table 3 were checked independently as to whether they meet the isolation conditions used in this paper. It turned out that only the galaxies UGC 3476, FG 202, UGC 3755, ESO 6-1, and KK 246 met these conditions fully. UGC 288 and And IV form a wide "pair" with a projected mutual distance of about $460 \mathrm{kpc}$. The galaxy IC 2038 forms a close pair with IC 2039 which is a member of the group NGC 1566. The galaxies KK 49 and Orion have a difference of $<100 \mathrm{~km} / \mathrm{s}$ in their radial velocities and have a projected distance on the order of $200 \mathrm{kpc}$. The peculiar loop shaped galaxy ESO 364-29 has close (up to $150 \mathrm{kpc}$ ) neighbors 
AM 0605-341 and NGC 2188 with mutual radial velocity differences $<50 \mathrm{~km} / \mathrm{s}$. ESO 489-56 has a rather high surface brightness and is at a distance of about $160 \mathrm{kpc}$ from ESO 490-17 (radial velocity difference $12 \mathrm{~km} / \mathrm{s}$ ). The galaxy KK 55 has a neighbor, NGC 2310 (radial velocity difference 321 $\mathrm{km} / \mathrm{s}$ and mutual separation $\sim 400 \mathrm{kpc}$ ), and can be regarded as isolated, the same can also be said of the galaxy KKH 46, which has a faint neighboring galaxy at a distance of $270 \mathrm{kpc}$. The galaxy ESO 558-11 has a neighbor, HIPASS J0705-20 (radial velocity difference $34 \mathrm{~km} / \mathrm{s}$, mutual separation $130 \mathrm{kpc}$ ), while DDO 47 is a member of a close pair with CGCG 37-33 (radial velocity difference 7 $\mathrm{km} / \mathrm{s}$, mutual distance $11 \mathrm{kpc}$ ). The galaxy ESO 564-30 can be regarded as sufficiently isolated; its nearest neighbor ESO 565-3 is located at a distance of $\sim 290 \mathrm{kpc}$ (velocity difference $61 \mathrm{~km} / \mathrm{s}$ ), and the bright galaxy NGC 2835 (velocity difference $118 \mathrm{~km} / \mathrm{s}$ ) is located at a distance of $\sim 450 \mathrm{kpc}$. We are providing this detailed comparison in order to demonstrate the arbitrariness of the criterion for selecting faint dwarf galaxies as "truly" isolated when the mutual spatial separations and spatial velocities of the galaxies are unknown.

\section{Discussion}

The procedure of clusterizing galaxies in the volume of the Local supercluster and its surroundings $\left(V_{L G}<3500 \mathrm{~km} / \mathrm{s}\right)$ using a stronger criterion of dynamic isolation has led to creation of the LOG catalog [11], which contains 520 galaxies at galactic latitudes $|b|>15^{\circ}$. Their relative number is only $4 \%$ of the $\mathrm{N}=10914$ galaxies in this volume. Most of the LOG-galaxies are normal spiral galaxies of late morphological types. If we choose only galaxies of types Ir, Im, Sm, and BCD ( T = $9,10)$ from this catalog, their total number is $\mathrm{N}(9,10)=87$. This subsample of isolated galaxies included 16 of the objects in Table 1: UGC 199, ESO 243-50, UGC 2352, KDG 32, HIJ0342+1808, UGC 3672, UGC 4117, KKSG 47, UGC 9875, KKR 21, KKR 26, KKR 30, KKR 34, KKR 75, UGC 12070, UGC 12771. Thus, about $20 \%$ of the dwarf galaxies simultaneously pass tests for physical isolation according to the two different isolation criteria. We believe this fraction is quite high. As a comparison, we note that a recent study [29] examined the effect of the surroundings on the HI and optical properties of 101 galaxies with absolute magnitudes $M_{r}-5 \log h_{70}>-16$ from the catalog [30] compiled from the SDSS survey data. The average absolute magnitude of the galaxies is $\left\langle M_{r}\right\rangle=-15.4$ and the average depth of their sample is $30 h_{70}^{-1} \mathrm{Mpc}$, both similar to our values. It was found [29] that $65 \%$ of the galaxies in this sample have no neighbors in the RC3 [31] with $M_{r}-5 \log h_{70}<-19$ at a projected distance of $500 h_{70} \mathrm{kpc}$ and a radial velocity difference within the range of $\pm 300 \mathrm{~km} / \mathrm{s}$. In this paper we have used a similar method for identifying isolated galaxies. Here the initial samples relied on various optical surveys. Furthermore, in [29] candidates were selected in terms of absolute magnitude, and in our work, mainly in terms of their morphological type and surface brightness. Probably, this is the case why there are no common objects in our list and in [29].

It should be noted that the isolated irregular galaxies in Table 1 do not differ significantly in size, luminosity, and amplitude of their internal motions from other galaxies of the same type in groups, or from the gas-rich dwarf galaxies examined in [32]. However, isolated late-type dwarf galaxies do differ significantly from non-isolated galaxies with an enhanced neutral hydrogen content per unit luminosity. Thus, in the Local volume, the population of dwarf galaxies detected in the HI line has a median value of $M(H I) / L_{B}=0.8$ in solar units, while the isolated objects in Table 1 have a median of $M(H I) / L_{B}=2.0$. Evidently, the absence of external tidal perturbations in the isolated 
dwarf galaxies does not favor the rapid expenditure of the gas contained in them on star formation processes.

The apparent magnitudes and surface brightnesses of the presumed isolated dSph galaxies are similar to those for spheroidal galaxies located in the nearby volume. So far, we are aware only of two cases of isolated dSph: KKR25 and APPLES1, which have distances of 1.9 and $8.5 \mathrm{Mpc}$ and absolute magnitudes $M_{B}=-9.9$ and -8.3 , respectively. The discovery of new dwarf galaxies populated only by old stars and lying beyond of groups and clusters regions, will be of fundamental interest for modern cosmological scenarios of galaxy formation.

In this paper we have used the DSS (http://archive.eso.org/dss) and SDSS (http://www.sdss.org) digital sky surveys, as well as the HYPERLEDA (http://leda.univlyon1.fr/) and NED (http://nedwww. ipac.caltech.edu) databases. This work was partially supported by grants from RFFI, No. 09-0290414-Ukr-f-a-i, MON Ukrainy, No. F28.2/059.

\section{REFERENCES}

1. Proceedings of IAU Conference "Galaxies in Isolation: Exploring Nature vs. Nurture", May 2009, Granada, Spain, ASP Conf. Ser. 421 (2010).

2. I. D. Karachentsev, V. E. Karachentseva, W. K. Huchtmeier, and D. I. Makarov, Astron. J. 127, 2031 (2004) (CNG).

3. F. Zwicky, E. Herzog, M. Karpowich, C. T. Kowal, and P. Wild (1961-1968), Catalogue of Galaxies and Clusters of Galaxies, California Institute of Technology, Pasadena, I-VI (CGCG).

4. P. Nilson, Uppsala General Catalogue of Galaxies, Uppsala Astron. Observ. 6, 1 (UGC) (1973).

5. E. Lauberts, The ESO/Uppsala Survey of the ESO (B) Atlas, Munich: ESO (1982).

6. V. E. Karachentseva, Soobshch. SAO 8, 3 (1973).

7. I. D. Karachentsev, Astron. Astrophys. Trans. 6, 1 (1994).

8. I. D. Karachentsev and D. I. Makarov, Astrophys. Bull. 63, 320 (2008).

9. D. I. Makarov and I. D. Karachentsev, Astrophys. Bull. 64, 24 (2008).

10. D. I. Makarov and I. D. Karachentsev, Mon. Notic. Roy. Astron. Soc. 412, 2498 (2011).

11. I. D. Karachentsev, D. I. Makarov, V. E. Karachentseva, and O. Y. Melnyk, Astrophys. Bull., 66, 1 (2011) (LOG) .

12. V. E. Karachentseva and M. E. Sharina, The Catalogue of low surface brightness dwarf galaxies, Comm. Spec. Astrophys. Obs. 57, 3 (1988).

13. B. Binggeli, M. Tarenghi, and A. R. Sandage, Astron. Astrophys. 228, 42 (1990) (BTS).

14. C. D. Impey, D. Sprayberry, M. J. Irwin, and C. D. Bothum, Astrophys. J. Suppl. Ser. 105, 209 (1996).

15. J. M. Schombert, R. A. Pildis, and J. A. Eder, Astrophys. J. Suppl. Ser. 111, 233 (1997).

16. A. B. Whiting, G. K. T. Hau, and M. Irwin, Astrophys. J. Suppl. Ser. 72, 245 (2002).

17. T-X. Thuan and P. O. Seitzer, Astrophys. J. 231, 237 (1979).

18. S. E. Schneider, T-X. Thuan, C. Magri, and J. E. Wadiak, Astrophys. J. Suppl. Ser. 72, 245 (1990).

19. V. E. Karachentseva and I. D. Karachentsev, Astron. Astrophys. Suppl. Ser. 127, 409 (1998) (KK).

20. V. E. Karachentseva, I. D. Karachentsev, and G. M. Richter, Astron. Astrophys. Suppl. Ser. 135, 221 (1999) (KKR). 
21. I. D. Karachentsev, V. E. Karachentseva, and W. K. Huchtmeier, Astron. Astrophys. 366, 428 (2001) (KKH).

22. V. E. Karachentseva and I. D. Karachentsev, Astron. Astrophys. Suppl. Ser. 146, 359 (2000) (KKs).

23. I. D. Karachentsev, V. E. Karachentseva, A. A. Suchkov, and E. K. Grebel, Astron. Astrophys. Suppl. Ser. 145, 415 (2000) (KKSG).

24. W. K. Huchtmeier, I. D. Karachentsev, and V. E. Karachentseva, Astron. Astrophys. 322, 375 (1997).

25. W. K. Huchtmeier, I. D. Karachentsev, V. E. Karachentseva, and M. Ehle, Astron. Astrophys. Suppl. Ser. 141, 469 (2000).

26. W.K.Huchtmeier, I. D. Karachentsev, and V. E. Karachentseva, Astron. Astrophys. 377, 801 (2001).

27. W.K.Huchtmeier, I. D. Karachentsev, and V. E. Karachentseva, Astron. Astrophys. 401, 483 (2003).

28. R. B. Tully, Nearby Galaxy Catalog, Cambridge University Press (1988).

29. M. Geha, M. R. Blanton, M. Masjedi, and A .A. West, Astrophys. J. 653, 240 (2006).

30. M. R. Blanton, D. J. Eisenshtein, D. V. Hogg, et al., Astrophys. J. 631, 208 (2005).

31. G. de Vaucouleurs, A. de Vaucouleurs, H. G. Corwin, et al., Third Reference Catalogue of Bright Galaxies, I-III, SpringerVerlag, Berlin, Heidelberg, New York (1991) (RC3).

32. R. F. Minchin, M. J. Disney, Q. A. Parker, et al., Mon. Notic. Roy. Astron. Soc. 355, 130 (2004). 